\title{
Outcomes of Metastatic Chromophobe Renal Cell Carcinoma (chrRCC) in the Targeted Therapy Era: Results from the International Metastatic Renal Cell Cancer Database Consortium (IMDC)
}

Steven M. Yip ${ }^{\mathrm{a}}$, Jose M. Ruiz Morales ${ }^{\mathrm{b}}$, Frede Donskov ${ }^{\mathrm{c}}$, Anna Fraccon ${ }^{\mathrm{d}}$, Umberto Basso ${ }^{\mathrm{e}}$, Brian I. Rini ${ }^{\mathrm{f}}$, Jae Lyun Lee ${ }^{\mathrm{g}}$, Georg A. Bjarnason ${ }^{\mathrm{h}}$, Hao-Wen Sim ${ }^{\mathrm{i}}$, Benoit Beuselinck ${ }^{\mathrm{j}}$, Ravindran Kanesvaran ${ }^{\mathrm{k}}$, James Brugarolas ${ }^{1}$, Kostas Koutsoukos ${ }^{\mathrm{m}}$, Simon Yuen Fai Fu ${ }^{\mathrm{n}}$, Takeshi Yuasa $^{\mathrm{o}}$, Ian Davis ${ }^{\mathrm{p}}$, Ajjai Alva ${ }^{\mathrm{q}}$, Christian Kollmannsberger ${ }^{\mathrm{r}}$, Toni K. Choueiri ${ }^{\mathrm{s}}$ and Daniel Y.C. Hengt,*

${ }^{a}$ Department of Medical Oncology, Tom Baker Cancer Centre, University of Calgary, Calgary, AB, Canada

${ }^{\mathrm{b}}$ Hospital Medica Sur, Mexico City, Mexico

${ }^{\mathrm{c}}$ Department of Oncology, Aarhus University Hospital, Aarhus, Denmark

${ }^{\mathrm{d}}$ Medical Oncology, Casa di Cura Pederzoli, Peschiera Del Garda, Peschiera Del Garda (VR), Italy

${ }^{\mathrm{e}}$ Instituto Oncologico Veneto, Venice, Italy

${ }^{\mathrm{f}}$ Cleveland Clinic Taussig Cancer Institute, Cleveland, OH, USA

${ }^{\mathrm{g}}$ Department of Oncology, Asan Medical Center, University of Ulsan College of Medicine, Seoul, South Korea

${ }^{\mathrm{h}}$ Sunnybrook Odette Cancer Centre, University of Toronto, Toronto, ON, Canada

${ }^{\mathrm{i}}$ Princess Margaret Cancer Centre, Toronto, ON, Canada

${ }^{\mathrm{j}}$ Department of General Medical Oncology, University Hospitals Leuven, Leuven, Belgium

${ }^{\mathrm{k}}$ National Cancer Centre Singapore, Singapore

${ }^{1}$ The University of Texas Southwestern Medical Center, Dallas, TX, USA

${ }^{\mathrm{m}}$ Department of Clinical Therapeutics, Oncology Unit, Alexandra Hospital, University of Athens, Athens, Greece

${ }^{\mathrm{n}}$ Auckland City Hospital, Auckland, New Zealand

${ }^{\circ}$ Department of Urology, Cancer Institute Hospital of Japanese Foundation for Cancer Research, Tokyo, Japan

${ }^{\mathrm{p}}$ The Institute of Cancer Research, The Royal Marsden NHS Foundation Trust, Sutton, UK

${ }^{\mathrm{q}}$ Department of Internal Medicine, Division of Hematology/Oncology, University of Michigan, Ann Arbor,

MI, USA

${ }^{\mathrm{r}}$ BC Cancer Agency, Vancouver Cancer Centre, Vancouver, BC, Canada

${ }^{\mathrm{s}}$ Dana-Farber/Brigham and Women's Cancer Center, Boston, MA, USA

${ }^{\mathrm{t}}$ Tom Baker Cancer Center, University of Calgary, Calgary, AB, Canada

\footnotetext{
*Correspondence to: Daniel Y.C. Heng, Tom Baker Cancer Center, University of Calgary, 133129 St NW, Calgary, AB, T2N 4N2, Canada. Tel.: +1 403521 3166; Fax: +1 403283 1651; E-mail: daniel.heng@ahs.ca.
} 
Background: Treatment outcomes are poorly characterized in patients with metastatic chromophobe renal cell cancer (chrRCC), a subtype of renal cell carcinoma.

Objective: This retrospective series aims to determine metastatic chrRCC treatment outcomes in the targeted therapy era. Methods: A retrospective data analysis was performed using the IMDC dataset of 4970 patients to determine metastatic chrRCC treatment outcomes in the targeted therapy era.

Results: $109 / 4970$ (2.2\%) patients had metastatic chrRCC out of all patients with mRCC treated with targeted therapy. These patients were compared with 4861/4970 (97.8\%) clear cell mRCC (ccRCC) patients. Patients with metastatic chrRCC had a similar OS compared to patients with ccRCC (23.8 months (95\% CI 16.7 - 28.1) vs 22.4 months (95\% CI 21.4 - 23.4), respectively $(p=0.0908)$. Patients with IMDC favorable $(18 \%)$, intermediate $(59 \%)$ and poor risk $(23 \%)$ had median overall survivals of $31.4,27.3$, and 4.8 months, respectively $(p=0.028)$.

Conclusions: To the authors' knowledge, this is the largest series of metastatic chrRCC patients and these results set new benchmarks for survival in clinical trial design and patient counseling. The IMDC criteria risk categories seem to stratify patients into appropriate favourable, intermediate, and poor risk groups, although larger patient numbers are required. It appears that outcomes between metastatic chrRCC and ccRCC are similar when treated with conventional targeted therapies. Patients with metastatic chrRCC can be treated with tyrosine kinase inhibitors and enrolled in clinical trials to further measure outcomes in this rare patient population.

Keywords: Metastatic, renal, chromophobe, international, targeted

\section{INTRODUCTION}

Renal cell carcinoma (RCC) is classified into clear cell and non-clear cell types. The commonest non-clear cell RCC subtypes include papillary, collecting duct, medullary and chromophobe, among the many subtypes identified by the 2016 World Health Organization and the 2012 International Society of Urological Pathology classification systems [1, 2]. Chromophobe renal cell carcinoma (chrRCC) is a rare subtype of renal cell carcinoma, which arises from the distal regions of the kidney, as opposed clear cell RCC (ccRCC), which arises from the proximal kidney.

Treatment outcomes are poorly characterized in patients with metastatic chromophobe renal cell cancer. This is largely a consequence of the rare manifestation of this subtype in a metastatic stage. ChrRCC occurs in $6-11 \%$ of all patients with RCC, as well as 6-7\% of all forms of metastatic renal cell carcinoma (mRCC) [3].

ChrRCC is typically associated with better prognosis, in comparison to ccRCC [3]. In a multiinstitutional retrospective case series of 291 patients with any stage chrRCCs, Volpe et al. reported that only $1.3 \%$ of chrRCC patients presented with distant metastases at the time of diagnosis [4]. The chrRCC 5 - and 10 -year cancer-specific survival were $93 \%$ and $88.9 \%$, respectively. Locally advanced or metastatic chrRCC, as well as those with sarcomatoid differentiation, were found to have the poorest outcomes. Pathological T4 stage (HR 75.9, $P=<0.001)$ and N/M stage (HR 21.5, $P<0.001$ ), as well as sarcomatoid differentiation (HR 25.9, $P=<0.001$ ) were significantly associated with CSS with univariable analysis.

To date, metastatic chrRCC outcomes have not yet been accurately studied in large numbers, particularly in the targeted therapy era. While the IMDC model (Heng Criteria) has been developed and validated in the the prognostication of patients with mRCC treated with targeted therapies, no prognostic tool has been specifically examined in this unique subtype population of mRCC [5]. To the author's knowledge, this is the largest analysis of metastatic chrRCC to date.

\section{OBJECTIVE}

This retrospective series aims to determine metastatic chrRCC treatment outcomes in the targeted therapy era. It also has the objective of examining the effectiveness of the IMDC model in predicting outcomes as a prognostic tool in the metastatic chrRCC subtype population.

\section{MATERIALS AND METHODS}

\section{Patient population and histology}

A retrospective data analysis was performed using the International mRCC Database Consortium (IMDC) dataset to characterize mRCC treatment outcomes in the targeted therapy era. Twenty-seven 
international cancer centres in Canada, the USA, Denmark, Greece, South Korea, Australia, New Zealand, Japan, Singapore, Belgium, and Italy provided consecutive patient data collected from hospital and pharmacy records using uniform database software and templates. Data were collected between 2005 and May 2016. Institutional review board approval was obtained from each participating centre.

All patients with metastatic ccRCC and chrRCC, who were treated with one or more lines of VEGF inhibitor (sunitinib, sorafenib, pazopanib, bevacizumab, axitinib) or mTOR targeted therapy (temsirolimus or everolimus), were included. Patients with mixed subtype RCC were excluded from analysis. Tumour histology was recorded using pathology reports generated by pathologists as part of routine patient diagnosis, prior to and independent of this study.

\section{Outcomes}

The IMDC collects demographic, baseline patient characteristics, and outcome data with each targeted therapy line administered. Overall survival (OS) was the primary outcome measured, and secondary outcomes - Time to Treatment Failure (TTF), and response rates (RR) - were calculated. OS was calculated from the time of initiation of targeted therapy to the time of death from any cause or censored at last follow up. Time to treatment failure (TTF) was defined as the time from initiation of targeted therapy to treatment discontinuation for any reason, including disease progression based upon Response Evaluation Criteria in Solid Tumors (RECIST) guidelines, treatment toxicity, cessation of therapy, any cause of death while on therapy, or censored at last follow up [6].

Median OS and TTF was reported and compared for the metastatic chrRCC and ccRCC patient groups. First-line targeted therapies were pooled separately to compare median OS rates between each type of therapy.

The International mRCC Database Consortium (IMDC) prognostic model was examined in metastatic chrRCC by categorizing metastatic chrRCC patients into IMDC risk groups using the following prognostic factors: hemoglobin below the lower limit of normal, corrected calcium greater than the upper limit of normal (ULN), neutrophils above ULN, platelets above ULN, Karnofsky performance status (KPS) below $80 \%$, and time from diagnosis to treatment of less than one year [3]. Patients are stratified into favorable, intermediate, and poor risk groups if they had none, 1 or 2 , and 3 or more prognostic factors, respectively.

\section{Statistical analysis}

SAS version 9.4 (Cary, NC, USA) was used to perform statistical analyses. Median OS and TTF was reported and compared for the metastatic chrRCC and ccRCC patient groups. OS of chrRCC and ccRCC was compared using Cox regression modeling, while adjusting for the individual IMDC prognostic factors. Kaplan-Meier curves were used to estimate median OS and TTF, and these outcomes were compared using the log rank test.

The best achieved RR was documented as complete response (CR), partial response (PR), stable disease (SD), and progressive disease (PD), based upon RECIST guidelines [6]. Overall response rate (ORR) was calculated by adding results meeting CR and PR criteria. Case deletion method was used when missing data was identified.

\section{RESULTS}

109/4970 (2.2\%) patients had metastatic chrRCC out of all patients with mRCC treated with targeted therapy. These patients were compared with 4861/4970 (97.8\%) metastatic ccRCC patients. Five patients with mixed chromophobe and clear cell mRCC were excluded from analysis. Baseline characteristics and risk factors are outlined and were similar to the normal patient population (Table 1).

Patients with metastatic chrRCC had a similar overall survival rate, as compared to patients with metastatic ccRCC (23.8 months (95\% CI 16.7 28.1 ) vs 22.4 months (95\% CI 21.4 - 23.4), respectively) (Fig. 1). Time to treatment failure was also similar amongst patients with metastatic chrRCC

Table 1

Baseline characteristics \& first-line therapy in metastatic chrRCC

\begin{tabular}{lc}
\hline $\begin{array}{l}\text { Baseline characteristics } \\
\& \text { first-line therapy }\end{array}$ & $\begin{array}{c}\text { Metastatic chrRCC patient } \\
\text { group }(N=109)\end{array}$ \\
\hline Gender & $59 / 109$ Male, 50/109 Female \\
Anemia & $50 \%(50 / 100)$ \\
Thrombocytosis & $15 \%(15 / 101)$ \\
Neutrophilia & $14 \%(14 / 100)$ \\
Karnofsky Performance Status $<80$ & $18 \%(18 / 101)$ \\
Less than 1 year from time of & $42 \%(46 / 109)$ \\
$\quad$ diagnosis to first-line therapy & \\
Prior Nephrectomy & $89 \%(97 / 109)$ \\
Brain metastases & $4 \%(4 / 101)$ \\
Greater than 1 site of metastasis & $73 \%(80 / 109)$ \\
\hline
\end{tabular}


Table 2

First line therapy of metastatic chrRCC patient group and respective overall survival

\begin{tabular}{ll}
\hline $\begin{array}{l}\text { First line therapy of metastatic } \\
\text { chrRCC patient group }\end{array}$ & Overall survival \\
\hline $\begin{array}{l}\text { Sunitinib 60\% (65/108) } \\
\text { Sorafenib } 15 \%(16 / 108)\end{array}$ & 21.1 mo (95\% CI 15.8 - 31.4) \\
Bevacizumab 0.9\% (1/109) & 25.7 mo (95\% CI 3.0 - 35.5) \\
Temsirolimus 10\% (11/109) & 18.4 mo (95\% CI N/A - N/A) \\
Pazopanib 10\% (11/109) & 29.4 mo (95\% 11.1 - NR) \\
Everolimus 3.7\% (4/109) & Median OS NR \\
& $(95 \%$ CI 4.8 - NR) \\
\hline
\end{tabular}

(6.9 months $(95 \% \mathrm{CI} 4.1-8.5))(N=109)$ and patients with ccRCC (7.6 (95\% CI $7.2-8.0) \quad(p=0.53)$ $(N=4861)$ (Fig. 2).

The overall response rate of patients with metastatic chrRCC to targeted therapy was $21 \%$ (4.2\% CR, 31.6\% PR, 47.4\% SD, 31.6\% PD) out of 95 patients with available response information.

In the first line of therapy, the majority of patients were treated with Sunitinib 60\% (65/108), followed by Sorafenib $15 \%$ (16/108), Temsirolimus $10 \%$ (11/109), Pazopanib 10\%(11/109), Everolimus 3.7\% (4/109), and then Bevacizumab 0.9\% (1/109); median OS rates were identified to be $21.1 \mathrm{mo}$ (95\% CI 15.8 - 31.4), 25.7 mo (95\% CI 3.0 - 35.5), 18.4 mo (95\% CI 1.1 - NR), 29.4 mo (95\% 11.9 - NR), NR (95\% CI 4.8 - NR), and 23.8 mo (95\% CI N/A - N/A), respectively (Table 2 ).

IMDC criteria risk category grouping of metastatic chrRCC was performed and revealed a significant difference between each group $(p=0.028)$. Patients with IMDC favorable ( 0 factors) $(N=15 / 82,18 \%)$, intermediate ( $1-2$ factors) $(N=48 / 82,59 \%)$ and poor risk (3 or more factors) $(N=19 / 82,23 \%)$ had median OS of 31.4, 27.3 and 4.8 months, respectively $(p=0.028)$ (Fig. 3). The IMDC criteria appear to stratify patients into appropriate favorable, intermediate, and poor risk groups [5].

\section{DISCUSSION}

To the authors' knowledge this is the largest series of metastatic chrRCC patients, and these results set new benchmarks for survival in clinical trial design and patient counseling.

ChrRCC generally portends a favorable prognosis in early stage RCC [3, 4]. Based upon previous data, it was expected that this would be applicable in metastatic chrRCC. However, previous data on metastatic chrRCC has been limited to small case series and phase II trials combining small samples of chrRCC patients with other non-clear cell mRCC patients. ESPN and ASPEN are both phase II trials that compared everolimus, a mammalian target of rapamycin (mTOR) inhibitor, and sunitinib, a vascular endothelial growth factor receptor (VEGFR) tyrosine kinase inhibitor (TKI), in non-clear cell $\mathrm{mRCC}$ patients. Prior to these trials, mTOR inhibitors and VEGFR-TKI's had yet to be prospectively compared in non-clear cell mRCC. ESPN included 12 metastatic chrRCC and reported a favorable median OS of 31.6 mo when treated with sunitinib and 25.1 mo with everolimus [7]. ORR were observed in $56 \%$ treated with sunitinib and 58\% treated with everolimus. ASPEN included 16 metastatic chrRCC and reported a PFS of 11.4 mo in metastatic chrRCC patients treated with everolimus $(95 \% \mathrm{CI}$ 5.7 - 19.4), compared to 5.5 mo in patients with metastatic chrRCC treated with sunitinib $(95 \% \mathrm{CI}$ 3.2 - 19.7) [8]. ORR of metastatic chrRCC patients was observed in $33 \%$ treated with everolimus, in contrast to $10 \%$ treated with sunitinib. This is postulated to be improved in everolimus, as chrRCC has activating mutations in PTEN-PI3K-mTOR pathway, which would result in an appropriate target for an mTOR inhibitor $[9,10]$.

However, both the ESPN and ASPEN results were limited by the small sample sizes of the metastatic chrRCC patients. Hence, their results are reported with large confidence intervals, and conclusions are difficult to make [7, 8]. Comparisons between these previous trials are not helpful, and they do not provide insight into outcomes, as compared with metastatic ccRCC patients.

Our retrospective analysis on the multiinstitutional IMDC dataset addresses these issues by gathering data from the largest international sampling of 109 patients with this rare subtype in the metastatic setting, which are compared and contrasted to the $4861 \mathrm{ccRCC}$ patient sample. Our results reveal that median OS and TTF outcomes observed in metastatic chrRCC and clear cell mRCC are similar when treated with targeted therapies. At an ORR of $21 \%$ in metastatic chrRCC treated with any targeted therapy, our ORR was lower than ESPN's reported rates. Since ORR was lower, outcomes in OS and TTF may possibly be a result of the relatively indolent natural evolution of this histological subtype.

The chrRCC patients were only $2.2 \%$ of the mRCC patients, in contrast to the estimated 6-7\% of all forms of $\mathrm{mRCC}$ in the general population [3]. This may be a result of a selection bias of chrRCC patients offered 


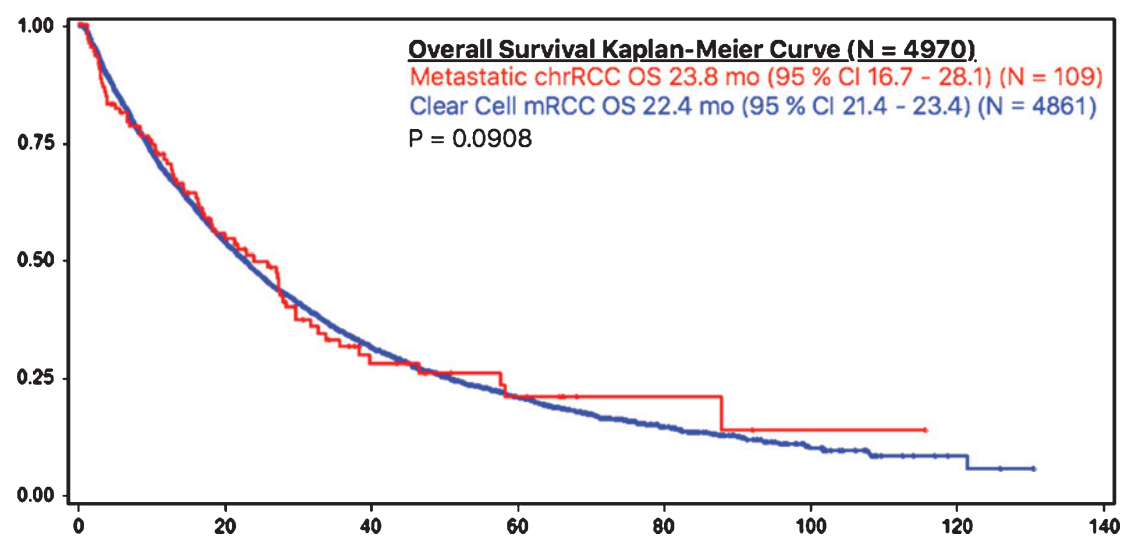

Fig. 1. OS in chrRCC vs clear cell mRCC.

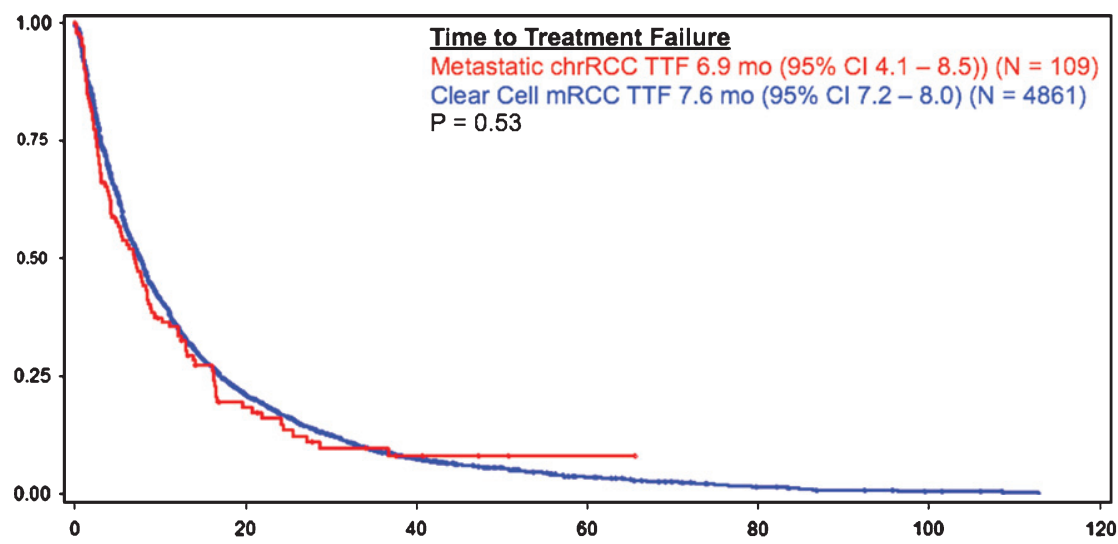

Fig. 2. TTF in chrRCC vs clear cell mRCC.

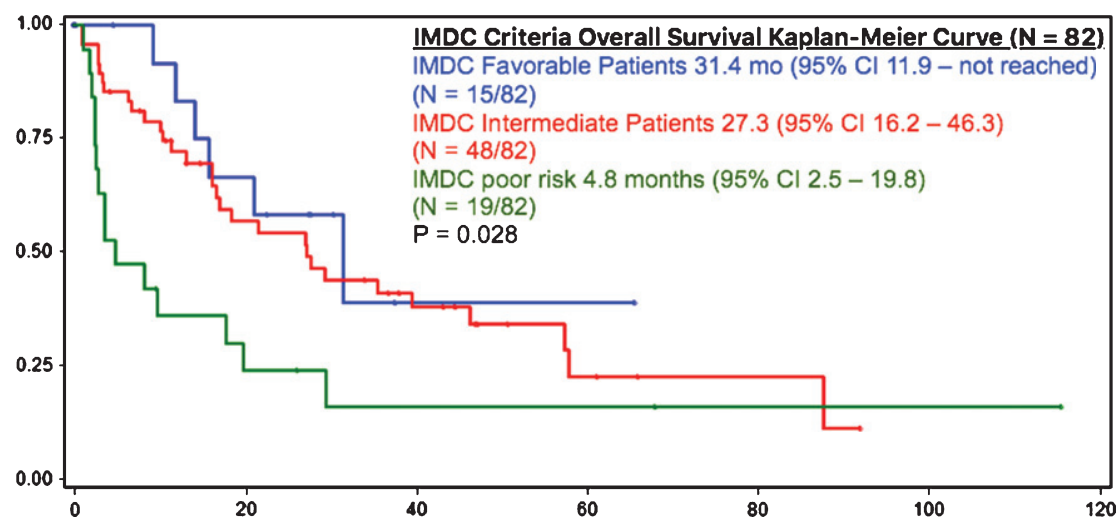

Fig. 3. OS in IMDC criteria risk categories.

targeted therapy. Although this study is limited by its small sample, retrospective design, and the absence of a central review, clinicians can now counsel patients with chromophobe mRCC on modern, standard of care therapeutic options. Patients can be offered tar- geted therapy knowing that efficacy and expected OS is similar to that of clear cell mRCC.

The IMDC model risk categories seem to stratify patients into appropriate favourable, intermediate, and poor risk groups. This is the largest series of 
metastatic chrRCC patients to date, but the smaller cohort size limits the definitive validation of the IMDC score in metastatic chrRCC. This raises the issue of examining rare diseases treated with targeted therapy, which requires a paradigm shift and a novel approach to trial design [11]. Although larger patient numbers should be examined in the future, the IMDC risk score can be cautiously applied in this patient population to counsel patients on prognosis and expected outcomes in the targeted therapy era.

This data also impacts future trial designs by setting survival standards for sample size calculations. Given the limited TTF and OS, patients with chrRCC should be enrolled in clinical trials to further improve outcomes in this rare patient population. The rarity of the disease will nevertheless be a challenge for such trials. Also, there is the disincentive to recruit rare subtypes, which may adversely influence the clarity of outcome results in common subtypes of mRCC, such as clear cell histology. Yet, future research will be required to examine bio-molecular nature of chrRCC, in order to develop novel targeted agents for the treatment of this rare mRCC subtype. Patients with chrRCC should continue to be enrolled in clinical trials to further measure outcomes in this rare patient population, as treatments advance in this rapidly evolving field.

\section{CONFLICT OF INTEREST}

Steven Yip,

COI: None

Jose Manuel Ruiz Morales

COI: Travel accommodations/expenses JanssenCilag, MSD Oncology, Bristol-Myers Squibb

Frede Donskov

COI: Research funding Novartis/Pfizer, GlaxoSmithKline

Anna Paola Fraccon

COI: None

Umberto Basso

COI: None

Brian I. Rini

COI: Consulting/advising Pfizer, Bristol-Myers

Squibb, GlaxoSmithKline, Novartis, AstraZeneca/ MedImmune
Travel accommodations/expenses Pfizer

Research funding Pfizer, Bristol-Myers Squibb, GlaxoSmithKline, Immatics, Roche/Genentech

Jae-Lyun Lee

COI: Consulting/advisory Astellas Pharma

Honoraria Pfizer, Astellas

Research funding Pfizer, Bayer, Janssen, Novartis, Exelixis

Georg A. Bjarnason

COI: None

Hao-Wen Sim

COI: Travel/Accommodations/Expenses Roche

Benoit Beuselinck

COI: Consulting/advisory Novartis

Speakers' bureau Novartis, GlaxoSmithKline, Pfizer

Honoraria Pfizer, Astellas

Research funding Pfizer, Novartis

Travel/expenses Pfizer

Ravindran Kanesvaran

COI: Honoraria Novartis, Bayer, Pfizer

Consulting/advisory Novartis, Bayer, Pfizer

James Brugarolas

COI: Consulting/advisory Bethyl Laboratories

Research funding/patents/royalties/other intellectual property Peloton Therapeutics

Konstantinos Koutsoukos

COI: None

Simon Yuen Fai Fu

COI: None

Takeshi Yuasa

COI: Expert testimony Astellas Pharma, Pfizer

Ian Davis

COI: None

Ajjai Shivaram Alva

COI: Consulting/advisory Eisai

Research funding Oncogenex, Genentech, Novartis, Bristol-Myers Squibb, BIND Biosciences

Christian K. Kollmannsberger

COI: Consulting/advisory Pfizer, Novartis, Seattle Genetics, Bristol-Myers Squibb 
Honoraria Novartis, Seattle Genetics, BristolMyers Squibb

Research funding Pfizer, Novartis

Toni K. Choueiri

COI: Consulting/advisory Novartis, Pfizer, Bayer, GlaxoSmithKline, Merck, Bristol-Myers Squibb, Roche/Genentech, Eisai, Prometheus, Foundation Medicine

Honoraria NCCN, UpToDate

Research funding Pfizer, Novartis, Merck, Exelixis, TRACON Pharma, GlaxoSmithKline, Bristol-Myers Squibb, AstraZeneca, Peloton Therapeutics, Roche/Genentech

\section{Daniel Yick Chin Heng}

COI: Consulting/advisory Novartis, Pfizer, Janssen, Astellas, Bristol-Myers Squibb

\section{REFERENCES}

[1] Moch H, Cubill AL, Humphrey PA, et al. The 2016 WHO Classification of Tumours of the Urinary System and Male Genital Organs-Part A: Renal, Penile, and Testicular Tumours. Eur Urol 2016;70(1):93-105.

[2] Delahunt B, Srigley JR, Montironi R, et al. Advances in renal neoplasia: Recommendations from the 2012
International Society of Urological Pathology Consensus Conference. Urol 2014;83(5):969-74.

[3] Reuter VE PJ. Contemporary approach to the classification of renal epithelial tumors. Semin Oncol 2000;27:124-137.

[4] Volpe A, Novara G, Antonelli A, et al. Chromophobe renal cell carcinoma (RCC): Oncological outcomes and prognostic factors in a large multicentre series. BJU International 2011;110:76-83.

[5] Heng DY, Xie W, Regan MM, et al. Prognostic factors for overall survival in patients with metastatic renal cell carcinoma treated with vascular endothelial growth factortargeted agents: Results from a large, multicenter study. J Clin Oncol 2009;27:5794-9.

[6] Eisenhauer EA, Therasse P, Bogaerts J, et al. New response evaluation criteria in solid tumours: Revised RECIST guideline (version 1.1). Eur J Cancer 2009;45(2):228-247. doi:10.1016/j.ejca.2008.10.026

[7] Tannir NM, Jonasch E, Albiges L et al. Everolimus versus sunitinib prospective evaluation in metastatic Non-Clear cell renal cell carcinoma (ESPN): A randomized multicenter phase 2 trial. European Urology 2015;69(5):866-874.

[8] Armstrong AJ, Halabi S, Eisen T, et al. Everolimus versus sunitinib for patients with metastatic non-clear cell renal cell carcinoma (ASPEN): A multicentre, open-label, randomised phase 2 trial. Lancet Oncol 2016;17(3):378-388.

[9] Chaux A, Albadine R, Schultz L, et al. Dysregulation of the mammalian target of rapamycin pathway in chromophobe renal cell carcinomas. Hum Pathol 2013;44:2323-2330.

[10] Davis CF, Ricketts CJ, Wang M, et al. The somatic genomic landscape of chromophobe renal cell carcinoma. Cancer Cell 2014;26:319-330.

[11] Porta C. How to identify active novel agents in rare cancers and then make them available: A need for a paradigm shift. Eur Urol 2012;62(6):1020-1. 